\title{
A prospective trial of stereotactic body radiation therapy for unresectable pancreatic cancer testing ablative doses
}

\author{
Stanley L. Liauw ${ }^{1}$, Lisa Ni ${ }^{1}$, Tianming $\mathrm{Wu}^{1}$, Fauzia Arif ${ }^{1}$, Denise Cloutier ${ }^{1}$, Mitchell C. Posner ${ }^{2}$, \\ Mark Kozloff ${ }^{3}$, Hedy L. Kindler ${ }^{3}$ \\ ${ }^{1}$ Department of Radiation Oncology, University of Chicago Medical Center, Chicago, IL, USA; ${ }^{2}$ Department of Surgical Oncology, University of \\ Chicago Medical Center, Chicago, IL, USA; ${ }^{3}$ Department of Medical Oncology, University of Chicago Medical Center, Chicago, IL, USA \\ Contributions: (I) Conception and design: SL Liauw, HL Kindler; (II) Administrative support: F Arif, D Cloutier; (III) Provision of study materials \\ or patients: SL Liauw; (IV) Collection and assembly of data: SL Liauw, L Ni; (V) Data analysis and interpretation: SL Liauw, L Ni, T Wu; (VI) \\ Manuscript writing: All authors; (VII) Final approval of manuscript: All authors. \\ Correspondence to: Stanley L. Liauw, MD. Department of Radiation Oncology, University of Chicago Medical Center, 5758 S Maryland Ave MC9006, \\ Chicago, IL 660637, USA. Email: sliauw@radonc.uchicago.edu.
}

Background: We explored the safety and efficacy of ablative doses of stereotactic body radiation therapy (SBRT) for unresectable pancreatic cancer.

Methods: This phase I/II trial included patients with unresectable pancreatic cancer previously treated with any number of cycles of induction chemotherapy. Patients were enrolled according to a $3+3$ dose escalation design at $10,12.5$, and $15 \mathrm{~Gy} \times 3$, with subsequent patients at the maximally tolerated dose (MTD). Treatment was delivered to gross tumor delineated with MRI fusion using image-guidance to fiducial markers. Dose-limiting toxicity (DLT) was defined as grade 3+ toxicity within 30 days. Secondary endpoints included late gastrointestinal (GI) toxicity, freedom from local failure (FFLF), and survival.

Results: Fifteen patients received a median 10 cycles of chemotherapy. There were no DLTs, and the MTD was 15 Gy $\times 3$. Thirty-day toxicity included grade 2 nausea (46\%) and grade 2 diarrhea (7\%). Median survival after SBRT was 12.8 months (23 months after diagnosis) and median relapse-free survival was 7 months. At 1 -year, FFLF was $80 \%$. Four patients had grade 3+ GI bleeding after 30 days (median 6 months). Grade 3+ GI bleeding was associated with tumor volume $(\mathrm{P}=0.01)$, heterogeneity of dose within the planning target volume (PTV) (V120, P=0.03), and duodenal dose (V26-30 Gy, P<0.2).

Conclusions: This aggressive SBRT regimen demonstrated limited 30-day morbidity, a moderate degree of local control, and a moderate risk for late GI bleeding. Further work is necessary to define the most appropriate hypofractionated radiation therapy (RT) regimen in the ablative dose range.

Keywords: Pancreatic cancer; radiation therapy (RT); stereotactic body radiation therapy (SBRT)

Submitted Apr 17, 2020. Accepted for publication Jul 31, 2020.

doi: 10.21037/jgo-20-187

View this article at: http://dx.doi.org/10.21037/jgo-20-187

\section{Introduction}

Pancreatic cancer is one of the most lethal cancers (1). In the United States, patients with a new diagnosis of pancreatic cancer have a 5 -year overall survival (OS) of $8 \%$ (2). Although the 5 -year survival is $32 \%$ for patients with localized disease (2), most patients present with locally advanced or metastatic disease at diagnosis and are not surgical candidates. Recent multi-agent chemotherapy regimens have significantly improved near-term survival in metastatic disease $(3,4)$. The vast majority of pancreatic cancer patients experience distant progression; however, a rapid autopsy series (5) demonstrated that $30 \%$ of deaths relate to local progression. Unfortunately, progress with local therapy has been more modest, as recent randomized 
trials testing conventionally fractioned radiation with concurrent chemotherapy for locally advanced disease have failed to improve survival $(6,7)$.

To address the need to improve local control in patients with unresectable disease, we studied whether ablative doses of stereotactic body radiation therapy (SBRT) could be safely administered after induction chemotherapy. SBRT, a form of radiation therapy (RT) which delivers large daily doses in 5 total fractions or less, has resulted in high rates of local control (8) across several types of cancer by providing a high biologically equivalent dose (BED) compared to conventionally fractionated radiation that is given over 5-6 weeks. A 3-fraction SBRT regimen using ablative doses in a phase II trial was reported in 2005 (9), but deemed unsafe due to severe toxicity within 2 weeks of treatment. We hypothesized that more modern imaging and delivery techniques could improve treatment tolerance of a 3 -fraction potentially ablative regimen (i.e., BED $>100)$ (10), and potentially enhance the therapeutic ratio of SBRT for unresectable pancreatic cancer. The primary goal of this phase I/II trial was to determine the maximally tolerated dose (MTD) of a 3 -fraction regimen, while secondary endpoints included local control and survival. A 3-fraction regimen was chosen in part to build off of an ongoing institutional trial of SBRT for oligometastases, with a predefined SBRT approach and normal tissue dose limits (11). We speculated that in a select cohort of patients without metastatic progression after induction chemotherapy, improved local control could favorably impact survival. We present the following article in accordance with the TREND reporting checklist (available at http://dx.doi.org/10.21037/jgo-20-187).

\section{Methods}

\section{Patients}

Eligible patients were 18 years of age or older and had histologically confirmed, unresected cancer of the pancreas or ampulla with any invasive histology, measurable radiographic disease by RECIST criteria (v1.1), or recurrent disease at the site of previous resection. Other inclusion criteria included Eastern Cooperative Oncology Group (ECOG) performance status of $\leq 2$, life expectancy of $\geq 3$ months, and absolute neutrophil count $\geq 1,800$ cells $/ \mathrm{mm}^{3}$, platelets $\geq 100,000$ cells $/ \mathrm{mm}^{3}$, hemoglobin $\geq 8.0 \mathrm{~g} / \mathrm{dL}$, creatinine $\leq 2 \times$ upper limit of normal, total bilirubin $\leq 2.5 \times$ upper limit of normal, and AST or ALT $\leq 2.5 \times$ upper limit of normal. Patients may have received any number of cycles of chemotherapy, but not within 2 weeks before the first fraction of RT, or 4 weeks after. Exclusion criteria were concurrent investigational therapy delivered over the period of treatment or up to 28 days post-radiation; prior RT to proposed area of treatment; pregnancy; primary disease $>7.5 \mathrm{~cm}$ in largest diameter on computed tomography (CT) or magnetic resonance imaging (MRI); gross extension of tumor into the lumen of duodenum; uncontrolled comorbidity including but not limited to active infection, symptomatic congestive heart failure, unstable angina, cardiac arrhythmia; or use of bevacizumab or vascular endothelial growth factor inhibitor within 3 months prior to or 6 months after RT.

\section{Study design}

This single center phase I/II trial followed a standard $3+3$ dose escalation paradigm. Patients received three fractions of SBRT starting at $10 \mathrm{~Gy} /$ fraction (total, $30 \mathrm{~Gy}$ ), increased by $2.5 \mathrm{~Gy} /$ fraction for each subsequent dose group if less than one-third of patients in each cohort experienced a dose-limiting toxicity (DLT). The MTD would be exceeded if $\geq 2$ of 6 experienced a DLT at that dose. DLT was defined by any grade $\geq 3$ gastrointestinal (GI) toxicity (including liver toxicity, symptomatic ascites, or enteritis), grade 4 nausea, or grade 4 skin toxicity within a 4-week window after treatment. Although it was appreciated that toxicity could occur beyond this window, this definition was chosen to minimize the required duration of observation and facilitate trial accrual, especially in absence of any well-defined peak onset of toxicity in a disease with expected median survival typically $<1$ year. An upper limit of 15 Gy/fraction (total dose, 45 Gy) was defined as the highest dose level. After establishment of the MTD, further patients would accrue at that dose. Patients were followed for a minimum of 1 year, and late grade 3+ GI toxicities occurring beyond 4 weeks were documented as a toxicity of interest which could influence future accrual. The study was conducted in accordance with the Declaration of Helsinki [2013] and was registered with ClinicalTrials.gov (NCT01342354). The study was approved by the institutional review board of the University of Chicago (IRB\# 16866B) and informed consent was taken from all the patients.

\section{Stereotactic body radiotherapy}

Prior to SBRT, all patients underwent placement of 3-5 gold fiducial markers (via 19-22 G endoscopic or 
percutaneous needle) in the pancreatic tumor, followed by non-gated magnetic resonance cholangiopancreatography (MRCP)/MRI with contrast of the abdomen $\geq 48$ hours later. During simulation, patients were immobilized supine with arms up. All patients underwent intravenous and oral contrast-enhanced CT-based simulation on a 64-slice Brilliance CT scanner (Brilliance CT, Big Bore, Philips Medical Systems, Andover, MA, USA). Respiratory gating was used to scan patients at end expiration. MRI images and the CT dataset were co-registered and reviewed with an attending radiologist to delineate gross tumor volume (GTV). A margin of 5-8 $\mathrm{mm}$ except for up to $12 \mathrm{~mm}$ in the craniocaudad dimension was used to create the planning target volume (PTV). Elective nodal regions were not included. The treatment technique included 9-15 field, coplanar, 3D-conformal RT (3DCRT, n=7) with intensity modulated RT (IMRT) or volumetric arc therapy $(\mathrm{n}=8)$ used if necessary to meet planning constraints. Normal dose constraints (Table S1) were derived from an ongoing institutional 3-fraction SBRT protocol for oligometastases (11). The stomach, duodenum, and jejunum/ileum had maximum point doses of $30 \mathrm{~Gy}$, with $<2 \mathrm{cc}$ of any of these structures receiving 24 Gy. Normal tissue constraints received priority over tumor volume coverage during plan optimization. Typical isodose lines are shown in Figure 1.

Treatment was given every other day with supervision by a physicist and was planned and supervised by one consistent attending radiation oncologist. Gated kilovoltage $(\mathrm{kV})$ orthogonal images and free breathing $\mathrm{kV}$ cone beam CT (CBCT) scans were acquired, prioritizing the intratumoral fiducial markers while also observing bowel location. Treatment was delivered with respiratory gating, with pre-treatment and mid-treatment on-board fluoroscopy to confirm fiducial marker position. Patients had no food for 2 hours prior to treatment and were prescribed a proton pump inhibitor, continuing for at least 4 weeks after SBRT. Dexamethasone was not prescribed.

\section{Response evaluation and follow-up}

Follow-up visits occurred 2 and 4 weeks after SBRT, then q2-3 months up to 12 months, and q6 months thereafter. Laboratory evaluations included blood count and platelets (at each clinical visit) and comprehensive metabolic panel and CA19-9 (every 6 months). In the reporting of freedom from local progression (FFLP), local control was defined according to RECIST criteria. Crude local control was also analyzed on the basis of clinical factors (e.g., uncontrolled pain at primary site, or progressive local disease in the absence of clear radiographic change). Acute and late toxicity was scored according to National Cancer Institute (NCI) Common Terminology Criteria for Adverse Events (CTCAE) v3.0.

\section{Statistical analysis}

This protocol was a phase I study that escalated dose according to determination of DLTs, in a $3+3$ design. The primary endpoint was to establish the MTD. The phase II portion of the study was designed to accrue 15 further patients at the putative MTD for a total enrollment of 24 patients. Secondary endpoints included FFLP, progression-free survival (PFS), and OS by the KaplanMeier method using JMP v14.0 (Cary, NC, USA). Freedom from failure analysis was calculated from start of SBRT to date of event while deaths or other events were censored.

\section{Results}

Between 2010 and 2016, 15 patients were accrued, including nine patients in the phase I portion of the study. The study was closed before target accrual due to slow accrual. Over this time period, roughly $40 \%$ of all referrals to radiation oncology for non-metastatic unresectable pancreatic cancer enrolled on this study; the most common reasons that patients were not enrolled included regional nodal disease or potential for future resection, for which hypofractionated radiotherapy with concurrent chemotherapy over 20 25 days was preferred. All patients had unresectable pancreatic cancer with invasive ductal histology. All patients were followed until death. Median follow-up for all patients was 13 months. Patient characteristics are described in Table 1. All patients had histologically proven adenocarcinoma of the pancreas, ECOG performance status $0-1$, and no overt metastatic disease. One patient $(7 \%)$ had recurrence in the resection bed after pancreaticoduodenectomy. Patients were treated with FOLFIRINOX $(n=12)$, gemcitabine $(n=2)$, or a combination of both $(n=1)$ for a median 10 cycles (range, $2-12$ ) of chemotherapy prior to SBRT. The median pretreatment CA19-9 was 108. The median GTV was 25.4 (range, 6.4-86.1) cc and median PTV was 76.0 (range, 47.9-162.5) cc. Prespecified normal tissue constraints were met in all cases, and the median volume receiving the prescription dose (V100) was 95\% to the GTV (range, 86-100\%) and 79\% (range, 66-94\%) to the PTV.

There were no DLTs observed within 4 weeks after 


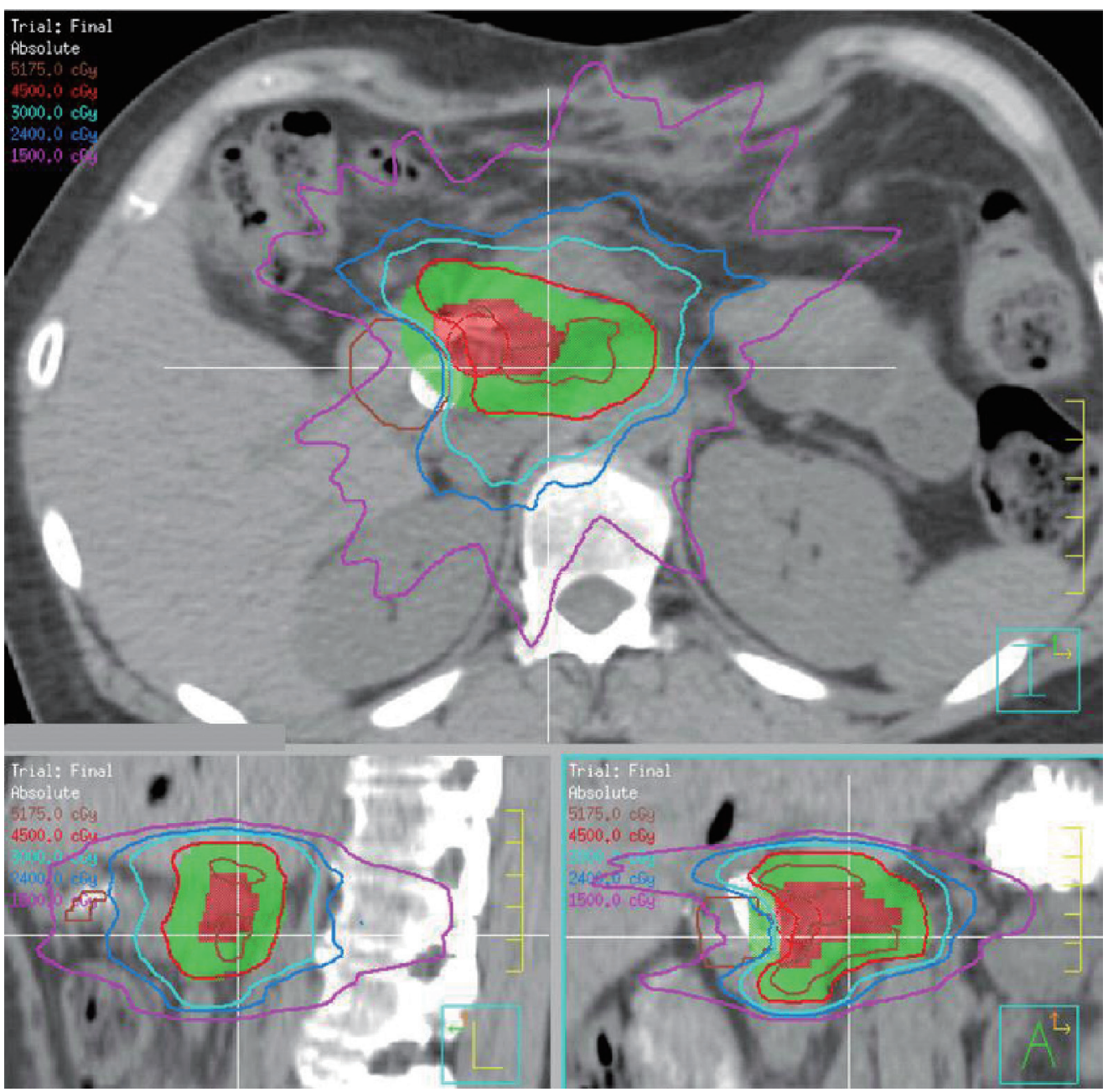

Figure 1 Example of a plan delivering 15 Gy $\times 3$ to the PTV (green colorwash). GTV (red colorwash) was 16 cc. Priority was given to achieve sparing goals to the duodenum (brown). The volume of PTV receiving the full prescription dose (V100) was $85 \%$; V100 GTV was $90 \%$. PTV, planning target volume; GTV, gross tumor volume.

SBRT. The MTD was defined as 15 Gy $\times 3$, which was adopted as the phase II dose. Three patients received $30 \mathrm{~Gy}$, 3 patients received $37.5 \mathrm{~Gy}$, and 9 patients received $45 \mathrm{~Gy}$. For the entire cohort, acute toxicity in the 4-week interval after SBRT included 1 patient with GI toxicity (grade 2, $\mathrm{n}=1,6 \%), 8$ patients $(54 \%)$ with nausea ( 1 with grade 1,7 with grade 2), and 5 patients (33\%) with fatigue (all grade 1). Acute hematologic toxicity was limited to 1 patient (7\%) with any grade 2 count (thrombocytopenia); otherwise 1 month post-SBRT there was 1 patient with grade 1 white blood cell counts (7\%), 11 patients with grade 1 hemoglobin counts $(73 \%)$, and 9 patients with grade $1-2$ platelet counts (60\%). When compared to pre-SBRT levels, a decrease in counts by grade occurred in hemoglobin in 3 patients $(20 \%$; median pre-treatment level 11.9, post-treatment level 12.3) and platelet count in 5 patients (33\%; median pre-treatment level 203, post-treatment level 148).

The median OS was 12.8 months after SBRT; the survival at 6-month, 1-year, and 3-year were $100 \%, 53 \%$, and $10 \%$, respectively (Figure $2 A$ ). The median survival after diagnosis was 23 (range, 9-95) months. Thirteen patients experienced disease progression after SBRT, including 4 with distant only, 2 with local only, and 7 with combined distant and local failure. The median PFS was 6.8 (range, 2-26) months; 1-year PFS was 33\% (Figure 2B). Median FFLP was 19.8 months; 1 -year FFLP was $80 \%$. The crude local control rate was $73 \%$ using RECIST criteria, but only $47 \%$ when incorporating relevant clinical information (e.g., 
Table 1 Patient and treatment characteristics

\begin{tabular}{|c|c|}
\hline Characteristics & Median [range or percentage] \\
\hline Age & 61 [34-81] \\
\hline \multicolumn{2}{|l|}{ Sex } \\
\hline Male & $6[40]$ \\
\hline Female & 9 [60] \\
\hline \multicolumn{2}{|l|}{ ECOG performance status } \\
\hline 0 & 8 [53] \\
\hline 1 & $7[47]$ \\
\hline \multicolumn{2}{|l|}{ Stage } \\
\hline T1N0 & $1[7]$ \\
\hline T3NO & $2[13]$ \\
\hline T4NO & $12[80]$ \\
\hline \multicolumn{2}{|l|}{ CA19-9 level pre-SBRT } \\
\hline Median [range], $\mathrm{ng} / \mathrm{mL}$ & $108[7-1,655]$ \\
\hline \multicolumn{2}{|l|}{ Pre-SBRT chemotherapy } \\
\hline Gemcitabine & $2[13]$ \\
\hline FOLFIRINOX & $11[73]$ \\
\hline $\begin{array}{l}\text { FOLFIRINOX, } \\
\text { gemcitabine }\end{array}$ & $1[7]$ \\
\hline FOLFIRINOX, FOLFOX & $1[7]$ \\
\hline \multicolumn{2}{|l|}{$\operatorname{GTV}\left(\mathrm{cm}^{3}\right)$} \\
\hline Median [range] & $25.4[6.4-86.1]$ \\
\hline \multicolumn{2}{|l|}{ PTV $\left(\mathrm{cm}^{3}\right)$} \\
\hline Median [range] & $76.0[47.9-162.5]$ \\
\hline V100 GTV (\%) & 94.7 [IQR: 90.5-98.7; range: 86-100] \\
\hline V100 PTV (\%) & 79.5 [IQR: 71.2-86.6; range: 66.5-93.7] \\
\hline
\end{tabular}

ECOG, Eastern Cooperative Oncology Group; SBRT, stereotactic body radiation therapy; GTV, gross tumor volume; PTV, planning target volume; IQR, interquartile range.

suspected local progression resulting in celiac plexus block). Median FFDM was 12 months; the 1 -year FFDM was $57 \%$. There was no correlation with SBRT dose received and FFLP $(\mathrm{P}=0.44)$ or OS $(\mathrm{P}=0.43)$. Local control was not associated with higher dose (crude local control $67 \%$ for dose $\leq 45$ Gy vs. $33 \%$ for dose 45 Gy, $\mathrm{P}=0.20$ ). The posttreatment CA19-9 decreased in all patients with elevated values prior to SBRT, from a median 108 to a median 23.5 (range, 3-379) at a median nadir of 5 months. Of the 8 patients who had pain prior to SBRT (median pain rating
2 on a scale of $1-10$; range, $1-5), 5(63 \%)$ had local pain response after SBRT.

A table of treatment parameters, acute toxicity, and late toxicity for all patients is shown in Table S2. Four patients had potentially related late grade 3+ GI bleeding toxicities ( 2 patients each treated with 30 and $45 \mathrm{~Gy}$ ), at a median of 6 months, including 1 grade 4 toxicity, and 1 grade 5 toxicity. Because of the possible contribution for uncontrolled local tumor to also result in GI bleed, these events did not change the MTD or trial accrual. The first two GI bleeding events (at the 30 Gy dose level) occurred after the 37.5 Gy dose level had already accrued with no DLT. All four events occurred at outside hospitals, making assignment of causality more difficult. We further studied the relationships of these GI bleed events and selected covariates under the assumption that they were treatment related, in exploratory analyses. Late grade 3+ GI bleeding was associated with GTV (as a continuous variable, $\mathrm{P}=0.09 ; 0 \%$ if $<25 \mathrm{cc}, 50 \%$ if $>25 \mathrm{cc}, \mathrm{P}=0.01$ ) as well as heterogeneity of dose within the tumor volume (PTV V120 as a continuous variable, $\mathrm{P}=0.03 ; 100 \%$ if PTV V120 $>15 \%$, vs. $8 \%$ if $<15 \%, \mathrm{P}<0.01$ ), and was weakly associated with increasing dose to the duodenum (V26-30 Gy, $\mathrm{P}<0.2)$. The patient with grade 4 bleeding underwent exploratory laparotomy 2 months after SBRT, but resection was aborted due to metastatic progression. The patient subsequently received immunotherapy on a phase I trial, and within 1 week had a duodenal bleed requiring blood transfusion and embolization. No further GI bleeding occurred over the next 9 months until death. The patient with grade 5 bleeding experienced a GI bleed to $\mathrm{Hg} 5.7$ without obvious source of bleeding by colonoscopy or initial esophagogastroduodenoscopy (EGD). Red blood cell (RBC) scan suggested a duodenal source of bleed, but repeat EGD showed post-RT changes without acute bleed. The patient underwent an unsuccessful attempt at embolization and ultimately experienced cardiopulmonary arrest in the setting of prior ischemic cardiomyopathy.

\section{Discussion}

In this phase I/II trial, we tested whether ablative doses of SBRT could be safely delivered in patients with unresectable pancreatic cancer after chemotherapy. The MTD of 15 Gy $\times 3$ was associated with minimal toxicity within 1 month after SBRT. With a median follow-up of 13 months, patients treated with 10-15 Gy $\times 3$ demonstrated a moderate to high rate of local control by imaging, and a median 
A

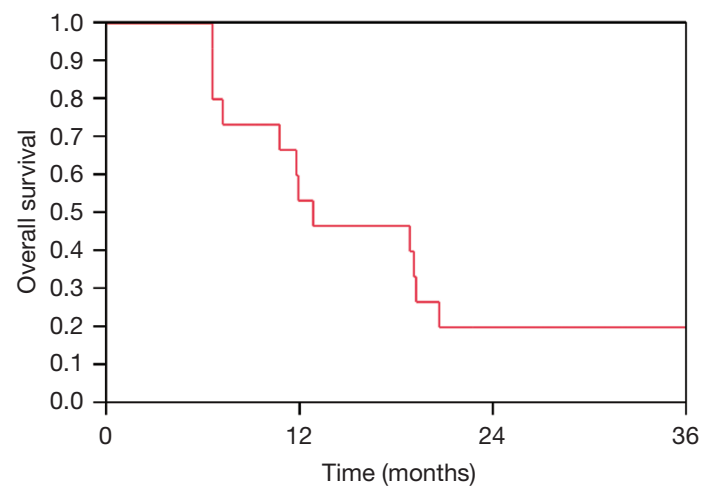

B

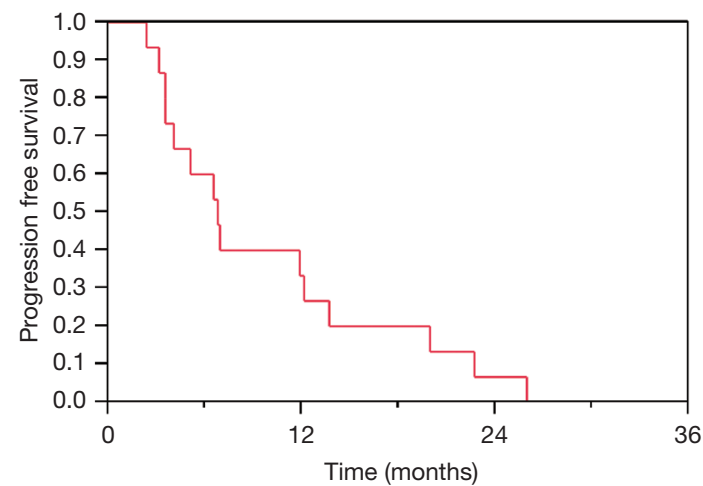

Figure 2 Overall survival (OS) (A) and progression-free survival (PFS) (B) after stereotactic body radiation therapy (SBRT).

survival time of 12.8 months after SBRT. However, the rate of late severe GI bleeding was not insignificant. Future use of this regimen should be carefully weighed in consideration of patient and tumor factors, in conjunction with utilization of strict duodenal sparing guidelines.

We were encouraged to achieve the maximal dose of 15 Gy $\times 3$ (BED 112.5 Gy, using $\alpha / \beta$ ratio of 10$)$ in a 1 -week course of therapy without meeting any DLT. The median PFS of $\sim 7$ months post-SBRT, and OS of 23 months postdiagnosis are better compared to historical controls (patients in LAP07 treated with chemoRT had a PFS of $~ 3$ months post-therapy with OS of 15.2 months after randomization), but it is unclear how much of this numerical improvement is primarily attributable to a well-selected patient population largely treated with more effective FOLFIRINOX chemotherapy. On the other hand, the study's longer term outcomes regarding local control and late toxicity could be considered disappointing. In distinction from reports of SBRT to other disease sites associated with local control rates exceeding $80-90 \%$, our crude local control rate for unresectable pancreatic cancer was $67 \%$ when using RECIST criteria, but only $~ 50 \%$ when considering both imaging and clinical information. Two hypotheses can be proposed which explain the lower than expected rate of local control. First, dose to the pancreatic tumor may have been compromised by setup error or motion, or by the requirement to meet dose sparing guidelines with priority over tumor coverage. In order to reduce the risk of a targeting miss, we utilized advanced methods of respiratory motion management (e.g., fluoroscopic confirmation of fiducial marker movement within the gated window), with relatively generous SBRT PTV margins $>5 \mathrm{~mm}$ on the gross tumor, which is larger than used in our current practice. Additionally, dose constraints used in this study were not as strict as modern constraints (12), in part because we did not want to overlook the chance for increased dose to improve control in a disease setting that is almost uniformly fatal. Nevertheless, respiratory motion management techniques may not fully protect against abdominal motion due to peristalsis (13) or tumor deformation (14), and the proximity of the duodenum typically compromised target coverage to meet normal tissue constraints (median V100 GTV 95\%, but V100 PTV only 80\%). Second, it remains entirely possible that pancreatic cancer is a radioresistant disease which may not be influenced by increasing radiation dose. Possible mechanisms of the ablative response of SBRT include small vessel destruction and adaptive immunity $(15,16)$, but this may be more problematic in the desmoplastic, immune suppressive environment (17) of pancreatic cancer post-chemotherapy. Favorable results using higher radiation doses (18) in a fractionated setting with concurrent chemotherapy [e.g., 67.5 Gy in 15 days (19)] have been reported and could potentially improve the therapeutic ratio $(10,20)$.

Although our study is limited by the small sample size and long accrual period of 6 years, our results are congruent with other prospective reports. The largest of these treated 49 patients with unresectable pancreatic cancer (21) to a dose of 33 Gy in 5 fractions (BED 1055 Gy). This regimen was associated with a $78 \%$ FFLP, median survival of 13.9 months from diagnosis, and $11 \%$ rate of late grade $2+$ toxicity. The lower rate of GI toxicity in this report was likely a result of the significantly lower equivalent dose. Several other regimens have also been tested, including 
25 Gy in 5 fractions with concurrent gemcitabine $\left(\mathrm{BED}_{10}\right.$ $37.5 \mathrm{~Gy})$ (22), $30 \mathrm{~Gy}$ in 3 fractions $\left(\mathrm{BED}_{10} 60 \mathrm{~Gy}\right)(23)$, 42-45 Gy in 6 fractions $\left(\mathrm{BED}_{10} 71-78\right)(24,25), 25$ Gy in 1 fraction $\left(\mathrm{BED}_{10} 87.5 \mathrm{~Gy}\right)(26)$, and 45 Gy in 3 fractions $\left(\mathrm{BED}_{10} 112.5\right)$ (9). All of these studies show similar rates of freedom from local failure (FFLF) in the 65-90\% range using RECIST criteria, with median survival 6-14 months from treatment, and $<5 \%$ risk of grade $3+$ toxicity with the exception of the studies testing $\mathrm{BED}_{10}>100$. The entirety of these trials suggests a lack of an easily identifiable dose response for unresectable disease treated with SBRT, and potentially increased risk of toxicity to normal tissues with the highest doses. The ablative dose regimen that was tested in our study led to a higher than expected rate of serious late GI toxicity, and future use of this regimen as planned is not recommended outside of a clinical protocol that evaluates toxicity up to 1 year out of therapy. Considering our study in the context of others, it would be reasonable to adjust the SBRT dose to meet an appropriate level of risk regarding normal tissue tolerance. The significant incidence of GI bleed and lack of a relationship between higher dose and local control in our study suggests the need to continue to prioritize duodenal sparing guidelines over dose when using SBRT to treat unresectable pancreatic cancer, and possibly use stricter normal tissue constraints such as a maximum duodenal dose of 24 Gy in 3 fractions (12). The use of a standard expansion on normal tissues may help to address variation in position that may have affected dose to these structures, and a planning organ at risk volume (PRV) margin on the duodenum is now employed in our clinic. The use of intrafraction monitoring with triggered $\mathrm{kV}$ images (27) or other means of accounting for setup variation [e.g., MR linac with adaptive real time planning $(28,29)]$ may justify tigher PTV margins. An optimistic view would be that continued improvements in targeting and motion management, and prolonged treatment over 5 fractions or more, will improve the therapeutic ratio of RT in the ablative dose range. This hypothesis is currently being tested in a number of clinical trials (including NCT03621644, NCT02454140).

The role of RT in unresectable pancreatic cancer treated with initial chemotherapy has been questioned with the randomized LAP07 study, because consolidative therapy in non-progressors did not improve survival. The result of this trial does not eliminate the value of consolidative local therapy (30), but it does force reconsideration of how we could improve upon conventional fractionated RT. Avenues of research to pursue include studying biomarkers (31) to personalize therapy, increasing the therapeutic ratio with novel regimens testing sensitizers or altered fractionation schemes, and improving our understanding of how to interpret post-treatment imaging to define surgical resectability (32). At best, a regimen that can substantially improve local control can improve survival when given to patients in whom the metastatic risk can be reduced with effective chemotherapy. If such an effective local therapy remains elusive, a convenient and well tolerated local regimen may at least offer patients a longer break from ongoing systemic therapy and an improved quality of life by reducing the risk of local progression. Further study is necessary to define the ideal patient population and optimal method of delivery of RT for patients with unresectable pancreatic cancer.

\section{Acknowledgments}

We would like to thank the patients and families who participated in this study. We acknowledge the AACR Vail Course which helped develop the trial.

Funding: This trial was internally funded.

\section{Footnote}

Reporting Checklist: The authors have completed the TREND reporting checklist. Available at http://dx.doi. org/10.21037/jgo-20-187

Data Sharing Statement: Available at http://dx.doi. org/10.21037/jgo-20-187

Conflicts of Interest: All authors have completed the ICMJE uniform disclosure form (available at http://dx.doi. org/10.21037/jgo-20-187). Dr. HLK reports grants and personal fees from Aduro, personal fees from Astellas, grants and personal fees from AstraZeneca, personal fees from Bayer, personal fees from Boehringer-Ingelheim, grants and personal fees from Bristol-Myers-Squibb, personal fees from Five Prime, grants and personal fees from GlaxoSimthKline, personal fees from Kyowa, grants and personal fees from Merck, personal fees from Paredox, grants and personal fees from Polaris, grants and personal fees from Lilly, grants and personal fees from Deciphera, grants and personal fees from Blueprint, grants and personal fees from Fibrogen, grants and personal fees from INHIBRx, grants and personal fees from Verastem, personal fees from Inventiva, outside the submitted work. 
Dr. MK reports personal fees from Roche, personal fees from Genentech, personal fees from AbbVie, personal fees from Celgene, personal fees from Merck, personal fees from Amgen, outside the submitted work. The other authors have no conflicts of interest to declare.

Ethical Statement: The authors are accountable for all aspects of the work in ensuring that questions related to the accuracy or integrity of any part of the work are appropriately investigated and resolved. The study was conducted in accordance with the Declaration of Helsinki (as was revised in 2013) and was registered with ClinicalTrials. gov (NCT01342354). The study was approved by the institutional review board of the University of Chicago (IRB\# 16866B) and informed consent was taken from all the patients.

Open Access Statement: This is an Open Access article distributed in accordance with the Creative Commons Attribution-NonCommercial-NoDerivs 4.0 International License (CC BY-NC-ND 4.0), which permits the noncommercial replication and distribution of the article with the strict proviso that no changes or edits are made and the original work is properly cited (including links to both the formal publication through the relevant DOI and the license). See: https://creativecommons.org/licenses/by-nc-nd/4.0/.

\section{References}

1. Torre LA, Bray F, Siegel RL, et al. Global cancer statistics, 2012. CA Cancer J Clin 2015;65:87-108.

2. Siegel RL, Miller KD, Jemal A. Cancer statistics, 2018. CA Cancer J Clin 2018;68:7-30.

3. Conroy T, Desseigne F, Ychou M, et al. FOLFIRINOX versus gemcitabine for metastatic pancreatic cancer. $\mathrm{N}$ Engl J Med 2011;364:1817-25.

4. Goldstein D, El-Maraghi RH, Hammel P, et al. nabPaclitaxel plus gemcitabine for metastatic pancreatic cancer: long-term survival from a phase III trial. J Natl Cancer Inst 2015;107:dju413.

5. Iacobuzio-Donahue CA, Fu B, Yachida S, et al. DPC4 gene status of the primary carcinoma correlates with patterns of failure in patients with pancreatic cancer. J Clin Oncol 2009;27:1806-13.

6. Hammel P, Huguet F, van Laethem JL, et al. Effect of chemoradiotherapy vs chemotherapy on survival in patients with locally advanced pancreatic cancer controlled after 4 months of gemcitabine with or without erlotinib: the
LAP07 randomized clinical trial. JAMA 2016;315:1844-53.

7. Barhoumi M, Mornex F, Bonnetain F, et al. Locally advanced unresectable pancreatic cancer: induction chemoradiotherapy followed by maintenance gemcitabine versus gemcitabine alone: definitive results of the 20002001 FFCD/SFRO phase III trial. Cancer Radiother 2011;15:182-91.

8. Timmerman RD, Herman J, Cho LC. Emergence of stereotactic body radiation therapy and its impact on current and future clinical practice. J Clin Oncol 2014;32:2847-54.

9. Hoyer M, Roed H, Sengelov L, et al. Phase-II study on stereotactic radiotherapy of locally advanced pancreatic carcinoma. Radiother Oncol 2005;76:48-53.

10. Crane CH, O'Reilly EM. Ablative radiotherapy doses for locally advanced: pancreatic cancer (LAPC). Cancer J 2017;23:350-4.

11. Salama JK, Chmura SJ, Mehta N, et al. An initial report of a radiation dose-escalation trial in patients with one to five sites of metastatic disease. Clin Cancer Res 2008;14:5255-9.

12. Pollom EL, Chin AL, Diehn M, et al. Normal tissue constraints for abdominal and thoracic stereotactic body radiotherapy. Semin Radiat Oncol 2017;27:197-208.

13. Mostafaei F, Tai A, Omari E, et al. Variations of MRIassessed peristaltic motions during radiation therapy. PLoS One 2018;13:e0205917.

14. Feng M, Balter JM, Normolle D, et al. Characterization of pancreatic tumor motion using cine MRI: surrogates for tumor position should be used with caution. Int J Radiat Oncol Biol Phys 2009;74:884-91.

15. Song CW, Kim MS, Cho LC, et al. Radiobiological basis of SBRT and SRS. Int J Clin Oncol 2014;19:570-8.

16. Popp I, Grosu AL, Niedermann G, et al. Immune modulation by hypofractionated stereotactic radiation therapy: therapeutic implications. Radiother Oncol 2016;120:185-94.

17. Dougan SK. The pancreatic cancer microenvironment. Cancer J 2017;23:321-5.

18. Golden DW, Novak CJ, Minsky BD, et al. Radiation dose $\geq 54$ Gy and CA 19-9 response are associated with improved survival for unresectable, non-metastatic pancreatic cancer treated with chemoradiation. Radiat Oncol 2012;7:156.

19. Krishnan S, Chadha AS, Suh Y, et al. Focal radiation therapy dose escalation improves overall survival in locally advanced pancreatic cancer patients receiving induction chemotherapy and consolidative chemoradiation. Int J 
Radiat Oncol Biol Phys 2016;94:755-65.

20. Reyngold M, Parikh P, Crane CH. Ablative radiation therapy for locally advanced pancreatic cancer: techniques and results. Radiat Oncol 2019;14:95.

21. Herman JM, Chang DT, Goodman KA, et al. Phase 2 multi-institutional trial evaluating gemcitabine and stereotactic body radiotherapy for patients with locally advanced unresectable pancreatic adenocarcinoma. Cancer 2015;121:1128-37.

22. Gurka MK, Kim C, He AR, et al. Stereotactic body radiation therapy (SBRT) combined with chemotherapy for unresected pancreatic adenocarcinoma. Am J Clin Oncol 2017;40:152-7.

23. Polistina F, Costantin G, Casamassima F, et al. Unresectable locally advanced pancreatic cancer: a multimodal treatment using neoadjuvant chemoradiotherapy (gemcitabine plus stereotactic radiosurgery) and subsequent surgical exploration. Ann Surg Oncol 2010;17:2092-101.

24. Mazzola R, Fersino S, Aiello D, et al. Linac-based stereotactic body radiation therapy for unresectable locally advanced pancreatic cancer: risk-adapted dose prescription and image-guided delivery. Strahlenther Onkol 2018;194:835-42.

25. Comito T, Cozzi L, Clerici E, et al. Can stereotactic body radiation therapy be a viable and efficient therapeutic option for unresectable locally advanced pancreatic adenocarcinoma? Results of a phase 2 study. Technol Cancer Res Treat 2017;16:295-301.

Cite this article as: Liauw SL, Ni L, Wu T, Arif F, Cloutier D, Posner MC, Kozloff M, Kindler HL. A prospective trial of stereotactic body radiation therapy for unresectable pancreatic cancer testing ablative doses. J Gastrointest Oncol 2020;11(6):13991407. doi: 10.21037/jgo-20-187
26. Schellenberg D, Kim J, Christman-Skieller C, et al. Single-fraction stereotactic body radiation therapy and sequential gemcitabine for the treatment of locally advanced pancreatic cancer. Int J Radiat Oncol Biol Phys 2011;81:181-8.

27. Korpics MC, Rokni M, Degnan M, et al. Utilizing the TrueBeam Advanced Imaging Package to monitor intrafraction motion with periodic $\mathrm{kV}$ imaging and automatic marker detection during VMAT prostate treatments. J Appl Clin Med Phys 2020;21:184-91.

28. Cusumano D, Boldrini L, Menna S, et al. Evaluation of a simplified optimizer for MR-guided adaptive RT in case of pancreatic cancer. J Appl Clin Med Phys 2019;20:20-30.

29. Henke L, Kashani R, Robinson C, et al. Phase I trial of stereotactic MR-guided online adaptive radiation therapy (SMART) for the treatment of oligometastatic or unresectable primary malignancies of the abdomen. Radiother Oncol 2018;126:519-26.

30. Balaban EP, Mangu PB, Khorana AA, et al. Locally advanced, unresectable pancreatic cancer: American Society of Clinical Oncology Clinical Practice Guideline. J Clin Oncol 2016;34:2654-68.

31. Loosen SH, Neumann UP, Trautwein C, et al. Current and future biomarkers for pancreatic adenocarcinoma. Tumour Biol 2017;39:1010428317692231.

32. Ferrone CR, Marchegiani G, Hong TS, et al. Radiological and surgical implications of neoadjuvant treatment with FOLFIRINOX for locally advanced and borderline resectable pancreatic cancer. Ann Surg 2015;261:12-7. 


\section{Supplementary}

Table S1 Normal tissue constraints for 3-fraction SBRT

\begin{tabular}{lcc}
\hline Organ & Volume & Dose \\
\hline Spinal cord & Any point & 21 Gy (7 Gy per fraction) \\
Esophagus & Any point & 27 Gy (9 Gy per fraction) \\
Stomach & $<2 \mathrm{cc}$ & 24 Gy (8 Gy per fraction) \\
& Any point & 30 Gy (10 Gy per fraction) \\
Heart/pericardium & Any point & 30 Gy (10 Gy per fraction) \\
Liver & $>700 \mathrm{cc}$ & $<17$ Gy (5.66 Gy per fraction) \\
Duodenum & $<2 \mathrm{cc}$ & 24 Gy (8 Gy per fraction) \\
& Any point & 30 Gy (10 Gy per fraction) \\
Jejunum/ileum & $<2 \mathrm{cc}$ & 24 Gy (8 Gy per fraction) \\
& Any point & 30 Gy (10 Gy per fraction) \\
Total kidney & $>200 \mathrm{cc}$ & $<15$ Gy (5 Gy per fraction) \\
\hline
\end{tabular}

SBRT, stereotactic body radiation therapy.

Table S2 Treatment parameters, toxicity, and disease outcomes for all patients

\begin{tabular}{|c|c|c|c|c|c|c|c|c|}
\hline Patient & $\begin{array}{c}\text { Induction } \\
\text { chemo-therapy }\end{array}$ & \multicolumn{2}{|r|}{ RT } & $\begin{array}{l}\text { Acute toxicity } \\
\text { within } 30 \text { days }\end{array}$ & $\begin{array}{l}\text { Late Gl } \\
\text { toxicity }\end{array}$ & \multicolumn{3}{|c|}{ Disease status (mo) } \\
\hline 1 & Gem $\times 2$ & 30 & $98.7,88.2$ & - & Grade 3 & 7 & 7 & 7 \\
\hline 2 & Gem ×12 & 30 & $99.7,93.7$ & Grade 1 nausea & Grade 3 & - & - & 12 \\
\hline 3 & $\mathrm{FFX} \times 5$ & 30 & $97.5,81.9$ & - & - & - & 22 & 95 \\
\hline 5 & FFX $\times 2$, Gem $\times 3$ & 37.5 & $95.2,70.8$ & Grade 2 nausea & - & - & 4 & 11 \\
\hline 6 & $\mathrm{FFX} \times 4$ & 37.5 & $86.0,66.5$ & Grade 2 nausea & - & 14 & 14 & 19 \\
\hline 7 & $\mathrm{FFX} \times 4$ & 45 & $92.6,82.1$ & Grade 2 nausea & - & 3 & - & 7 \\
\hline 8 & FFX $\times 12$ & 45 & $97.2,75.5$ & Grade 2 nausea & - & 29 & 12 & 37 \\
\hline 11 & FFX $\times 12$ & 45 & $89.1,74.4$ & Grade 2 GI & Grade 4 & - & 2 & 12 \\
\hline 12 & FFX $\times 1$, FOLFOX $\times 6$ & 45 & $90.9,71.2$ & Grade 2 nausea & - & 5 & 5 & 6 \\
\hline 13 & FFX $\times 12$ & 45 & $100,87.8$ & - & - & 19 & 47 & 50 \\
\hline 14 & $\mathrm{FFX} \times 4$ & 45 & $90.5,67.0$ & - & Grade 5 & - & - & 7 \\
\hline 15 & $\mathrm{FFX} \times 12$ & 45 & $90.4,84.6$ & Grade 2 nausea & - & 19 & - & 21 \\
\hline
\end{tabular}

V100\% indicates the percent volume of the GTV or PTV which received the full prescription dose. RT, radiation therapy; GTV, gross tumor volume; PTV, planning target volume; Gl, gastrointestinal; mo, months; Gem, gemcitabine; FFX, FOLFIRINOX. 